\title{
Limits on a muon flux from Kaluza-Klein dark matter annihilations in the Sun from the IceCube 22-string detector
}

R. Abbasi,${ }^{26}$ Y. Abdou, ${ }^{20}$ T. Abu-Zayyad,${ }^{31}$ J. Adams, ${ }^{15}$ J. A. Aguilar ${ }^{26}$ M. Ahlers,${ }^{30}$ K. Andeen, ${ }^{26}$ J. Auffenberg,${ }^{37}$ X. Bai, ${ }^{29}$ M. Baker, ${ }^{26}$ S. W. Barwick, ${ }^{22}$ R. Bay, ${ }^{7}$ J. L. Bazo Alba, ${ }^{38}$ K. Beattie, ${ }^{8}$ J. J. Beatty, ${ }^{17,18}$ S. Bechet, ${ }^{12}$ J. K. Becker, ${ }^{10}$ K.-H. Becker, ${ }^{37}$ M. L. Benabderrahmane, ${ }^{38}$ J. Berdermann,${ }^{38}$ P. Berghaus, ${ }^{26}$ D. Berley, ${ }^{16}$ E. Bernardini,${ }^{38}$ D. Bertrand, ${ }^{12}$ D. Z. Besson, ${ }^{24}$ M. Bissok, ${ }^{1}$ E. Blaufuss,${ }^{16}$ D. J. Boersma, ${ }^{1}$ C. Bohm, ${ }^{32}$ J. Bolmont,${ }^{38}$ O. Botner,${ }^{35}$ L. Bradley, ${ }^{34}$ J. Braun, ${ }^{26}$ D. Breder,${ }^{37}$ M. Carson, ${ }^{20}$ T. Castermans, ${ }^{28}$ D. Chirkin, ${ }^{26}$ B. Christy, ${ }^{16}$ J. Clem, ${ }^{29}$ S. Cohen, ${ }^{23}$ D. F. Cowen,${ }^{34,33}$ M. V. D’Agostino, ${ }^{7}$ M. Danninger,${ }^{32, *}$ C. T. Day,${ }^{8}$ C. De Clercq,${ }^{13}$ L. Demirörs,${ }^{23}$ O. Depaepe, ${ }^{13}$ F. Descamps,${ }^{20}$ P. Desiati, ${ }^{26}$ G. de Vries-Uiterweerd, ${ }^{20}$ T. DeYoung, ${ }^{34}$ J. C. Díaz-Vélez, ${ }^{26}$ J. Dreyer,,${ }^{19,10}$ J. P. Dumm, ${ }^{26}$ M. R. Duvoort, ${ }^{36}$ W. R. Edwards, ${ }^{8}$ R. Ehrlich, ${ }^{16}$ J. Eisch,${ }^{26}$ R. W. Ellsworth,${ }^{16}$ O. Engdegård, ${ }^{35}$ S. Euler, ${ }^{1}$ P. A. Evenson, ${ }^{29}$ O. Fadiran, ${ }^{4}$ A. R. Fazely, ${ }^{6}$ T. Feusels, ${ }^{20}$ K. Filimonov, ${ }^{7}$ C. Finley, ${ }^{32}$ M. M. Foerster, ${ }^{34}$ B. D. Fox,${ }^{34}$ A. Franckowiak, ${ }^{9}$ R. Franke, ${ }^{38}$ T. K. Gaisser, ${ }^{29}$ J. Gallagher ${ }^{25}$ R. Ganugapati, ${ }^{26}$ L. Gerhardt,,${ }^{8,7}$ L. Gladstone, ${ }^{26}$ A. Goldschmidt, ${ }^{8}$ J. A. Goodman, ${ }^{16}$

R. Gozzini, ${ }^{27}$ D. Grant, ${ }^{34}$ T. Griesel, ${ }^{27}$ A. Groß, ${ }^{15,21}$ S. Grullon, ${ }^{26}$ R. M. Gunasingha, ${ }^{6}$ M. Gurtner, ${ }^{37} \mathrm{C}$. Ha,${ }^{34}$

A. Hallgren, ${ }^{35}$ F. Halzen, ${ }^{26}$ K. Han,${ }^{15}$ K. Hanson, ${ }^{26}$ Y. Hasegawa, ${ }^{14}$ K. Helbing, ${ }^{37}$ P. Herquet,${ }^{28}$ S. Hickford, ${ }^{15}$ G. C. Hill ${ }^{26}$ K. D. Hoffman, ${ }^{16}$ A. Homeier, ${ }^{9}$ K. Hoshina, ${ }^{26}$ D. Hubert, ${ }^{13}$ W. Huelsnitz, ${ }^{16}$ J.-P. Hülß, ${ }^{1}$ P. O. Hulth, ${ }^{32}$ K. Hultqvist, ${ }^{32}$ S. Hussain, ${ }^{29}$ R. L. Imlay, ${ }^{6}$ M. Inaba, ${ }^{14}$ A. Ishihara,${ }^{14}$ J. Jacobsen,${ }^{26}$ G. S. Japaridze, ${ }^{4}$ H. Johansson, ${ }^{32}$ J. M. Joseph, ${ }^{8}$ K.-H. Kampert, ${ }^{37}$ A. Kappes, ${ }^{26, \dagger}$ T. Karg, ${ }^{37}$ A. Karle, ${ }^{26}$ J. L. Kelley, ${ }^{26}$ N. Kemming, ${ }^{9}$ P. Kenny, ${ }^{24}$ J. Kiryluk, ${ }^{8,7}$ F. Kislat,${ }^{38}$ S. R. Klein ${ }^{8,7}$ S. Knops, ${ }^{1}$ G. Kohnen, ${ }^{28}$ H. Kolanoski, ${ }^{9}$ L. Köpke, ${ }^{27}$ D. J. Koskinen, ${ }^{34}$ M. Kowalski, ${ }^{11}$ T. Kowarik, ${ }^{27}$ M. Krasberg, ${ }^{26}$ T. Krings, ${ }^{1}$ G. Kroll,${ }^{27}$ K. Kuehn, ${ }^{17}$ T. Kuwabara, ${ }^{29}$ M. Labare, ${ }^{12}$ S. Lafebre, ${ }^{34}$ K. Laihem, ${ }^{1}$ H. Landsman, ${ }^{26}$ R. Lauer, ${ }^{38}$ R. Lehmann, ${ }^{9}$ D. Lennarz, ${ }^{1}$ A. Lucke, ${ }^{9}$ J. Lundberg, ${ }^{35}$ J. Lünemann, ${ }^{27}$ J. Madsen, ${ }^{31}$ P. Majumdar, ${ }^{38}$ R. Maruyama, ${ }^{26}$ K. Mase, ${ }^{14}$ H. S. Matis, ${ }^{8}$ C. P. McParland,${ }^{8}$ K. Meagher, ${ }^{16}$ M. Merck, ${ }^{26}$ P. Mészáros,${ }^{33,34}$ T. Meures, ${ }^{1}$ E. Middell, ${ }^{38}$ N. Milke, ${ }^{19}$ H. Miyamoto, ${ }^{14}$ T. Montaruli, ${ }^{26, \$}$ R. Morse, ${ }^{26}$ S. M. Movit,${ }^{33}$ R. Nahnhauer, ${ }^{38}$ J. W. Nam, ${ }^{22}$ P. Nießen, ${ }^{29}$ D. R. Nygren, ${ }^{8}$ S. Odrowski, ${ }^{21}$ A. Olivas, ${ }^{16}$ M. Olivo, ${ }^{35,10}$ M. Ono, ${ }^{14}$ S. Panknin, ${ }^{9}$ S. Patton, ${ }^{8}$ L. Paul, ${ }^{1}$ C. Pérez de los Heros,${ }^{35}$ J. Petrovic, ${ }^{12}$ A. Piegsa, ${ }^{27}$ D. Pieloth,${ }^{19}$ A. C. Pohl,${ }^{35,}{ }^{8}$ R. Porrata, ${ }^{7}$ N. Potthoff, ${ }^{37}$ P. B. Price, ${ }^{7}$ M. Prikockis ${ }^{34}$ G. T. Przybylski, ${ }^{8}$ K. Rawlins, ${ }^{3}$ P. Redl, ${ }^{16}$ E. Resconi,${ }^{21}$ W. Rhode,,${ }^{19}$ M. Ribordy, ${ }^{23}$ A. Rizzo, ${ }^{13}$ J. P. Rodrigues, ${ }^{26}$ P. Roth, ${ }^{16}$ F. Rothmaier, ${ }^{27}$ C. Rott, ${ }^{17}$ C. Roucelle, ${ }^{21}$ D. Rutledge, ${ }^{34}$ B. Ruzybayev, ${ }^{29}$ D. Ryckbosch,${ }^{20}$ H.-G. Sander, ${ }^{27}$ S. Sarkar, ${ }^{30}$ K. Schatto, ${ }^{27}$ S. Schlenstedt ${ }^{38}$ T. Schmidt, ${ }^{16}$ D. Schneider, ${ }^{26}$ A. Schukraft, ${ }^{1}$ O. Schulz, ${ }^{21}$ M. Schunck, ${ }^{1}$ D. Seckel, ${ }^{29}$ B. Semburg,${ }^{37}$ S. H. Seo, ${ }^{32}$ Y. Sestayo, ${ }^{21}$ S. Seunarine, ${ }^{15}$ A. Silvestri, ${ }^{22}$ A. Slipak,${ }^{34}$ G. M. Spiczak ${ }^{31}$ C. Spiering, ${ }^{38}$ M. Stamatikos, ${ }^{17}$ T. Stanev,${ }^{29}$ G. Stephens, ${ }^{34}$ T. Stezelberger,${ }^{8}$ R. G. Stokstad,${ }^{8}$ M. C. Stoufer, ${ }^{8}$ S. Stoyanov, ${ }^{29}$ E. A. Strahler, ${ }^{26}$ T. Straszheim, ${ }^{16}$ K.-H. Sulanke, ${ }^{38}$ G. W. Sullivan, ${ }^{16}$ Q. Swillens, ${ }^{12}$ I. Taboada, ${ }^{5}$ A. Tamburro, ${ }^{31}$ O. Tarasova, ${ }^{38}$ A. Tepe,${ }^{37} \mathrm{~S}$. Ter-Antonyan, ${ }^{6} \mathrm{C}$. Terranova, ${ }^{23} \mathrm{~S}$. Tilav, ${ }^{29} \mathrm{P}$. A. Toale, ${ }^{34}$ J. Tooker, ${ }^{5}$ D. Tosi,${ }^{38}$ D. Turčan, ${ }^{16}$ N. van Eijndhoven, ${ }^{13}$ J. Vandenbroucke, ${ }^{7}$ A. Van Overloop, ${ }^{20}$ J. van Santen, ${ }^{9}$ B. Voigt,${ }^{38}$ C. Walck, ${ }^{32}$ T. Waldenmaier, ${ }^{9}$ M. Wallraff, ${ }^{1}$ M. Walter ${ }^{38}$ C. Wendt, ${ }^{26}$ S. Westerhoff, ${ }^{26}$ N. Whitehorn, ${ }^{26}$ K. Wiebe, ${ }^{27}$ C. H. Wiebusch, ${ }^{1}$ A. Wiedemann, ${ }^{19}$ G. Wikström, ${ }^{32, \|}$ D. R. Williams, ${ }^{2}$ R. Wischnewski, ${ }^{38}$ H. Wissing, ${ }^{16}$ K. Woschnagg, ${ }^{7}$ C. Xu, ${ }^{29}$ X. W. Xu, ${ }^{6}$ G. Yodh, ${ }^{22}$ and S. Yoshida ${ }^{14}$

\section{(IceCube Collaboration)}

${ }^{1}$ III. Physikalisches Institut, RWTH Aachen University, D-52056 Aachen, Germany

${ }^{2}$ Department of Physics and Astronomy, University of Alabama, Tuscaloosa, Alabama 35487, USA

${ }^{3}$ Department of Physics and Astronomy, University of Alaska Anchorage, 3211 Providence Drive, Anchorage, Alaska 99508, USA ${ }^{4}$ CTSPS, Clark-Atlanta University, Atlanta, Georgia 30314, USA

${ }^{5}$ School of Physics and Center for Relativistic Astrophysics, Georgia Institute of Technology, Atlanta, Georgia 30332, USA

${ }^{6}$ Department of Physics, Southern University, Baton Rouge, Louisiana 70813, USA

${ }^{7}$ Department of Physics, University of California, Berkeley, California 94720, USA

${ }^{8}$ Lawrence Berkeley National Laboratory, Berkeley, California 94720, USA

${ }^{9}$ Institut für Physik, Humboldt-Universität zu Berlin, D-12489 Berlin, Germany

${ }^{10}$ Fakultät für Physik \& Astronomie, Ruhr-Universität Bochum, D-44780 Bochum, Germany

${ }^{11}$ Physikalisches Institut, Universität Bonn, Nussallee 12, D-53115 Bonn, Germany

${ }^{12}$ Université Libre de Bruxelles, Science Faculty CP230, B-1050 Brussels, Belgium

${ }^{13}$ Vrije Universiteit Brussel, Dienst ELEM, B-1050 Brussels, Belgium

${ }^{14}$ Department of Physics, Chiba University, Chiba 263-8522, Japan 
${ }^{15}$ Department of Physics and Astronomy, University of Canterbury, Private Bag 4800, Christchurch, New Zealand

${ }^{16}$ Department of Physics, University of Maryland, College Park, Maryland 20742, USA

${ }^{17}$ Department of Physics and Center for Cosmology and Astro-Particle Physics, Ohio State University, Columbus, Ohio 43210, USA

${ }^{18}$ Department of Astronomy, Ohio State University, Columbus, Ohio 43210, USA

${ }^{19}$ Department of Physics, TU Dortmund University, D-44221 Dortmund, Germany

${ }^{20}$ Department of Subatomic and Radiation Physics, University of Gent, B-9000 Gent, Belgium

${ }^{21}$ Max-Planck-Institut für Kernphysik, D-69177 Heidelberg, Germany

${ }^{22}$ Department of Physics and Astronomy, University of California, Irvine, California 92697, USA

${ }^{23}$ Laboratory for High Energy Physics, École Polytechnique Fédérale, CH-1015 Lausanne, Switzerland

${ }^{24}$ Department of Physics and Astronomy, University of Kansas, Lawrence, Kansas 66045, USA

${ }^{25}$ Department of Astronomy, University of Wisconsin, Madison, Wisconsin 53706, USA

${ }^{26}$ Department of Physics, University of Wisconsin, Madison, Wisconsin 53706, USA

${ }^{27}$ Institute of Physics, University of Mainz, Staudinger Weg 7, D-55099 Mainz, Germany

${ }^{28}$ University of Mons-Hainaut, 7000 Mons, Belgium

${ }^{29}$ Bartol Research Institute and Department of Physics and Astronomy, University of Delaware, Newark, Delaware 19716, USA

${ }^{30}$ Department of Physics, University of Oxford, 1 Keble Road, Oxford OX1 3NP, UK

${ }^{31}$ Department of Physics, University of Wisconsin, River Falls, Wisconsin 54022, USA

${ }^{32}$ Oskar Klein Centre and Department of Physics, Stockholm University, SE-10691 Stockholm, Sweden

${ }^{33}$ Department of Astronomy and Astrophysics, Pennsylvania State University, University Park, Pennsylvania 16802, USA

${ }^{34}$ Department of Physics, Pennsylvania State University, University Park, Pennsylvania 16802, USA

${ }^{35}$ Department of Physics and Astronomy, Uppsala University, Box 516, S-75120 Uppsala, Sweden

${ }^{36}$ Department of Physics and Astronomy, Utrecht University/SRON, NL-3584 CC Utrecht, The Netherlands

${ }^{37}$ Department of Physics, University of Wuppertal, D-42119 Wuppertal, Germany

${ }^{38}$ DESY, D-15735 Zeuthen, Germany

(Received 23 October 2009; published 29 March 2010)

A search for muon neutrinos from Kaluza-Klein dark matter annihilations in the Sun has been performed with the 22-string configuration of the IceCube neutrino detector using data collected in 104.3 days of live time in 2007. No excess over the expected atmospheric background has been observed. Upper limits have been obtained on the annihilation rate of captured lightest Kaluza-Klein particle (LKP) WIMPs in the Sun and converted to limits on the LKP-proton cross sections for LKP masses in the range 250-3000 GeV. These results are the most stringent limits to date on LKP annihilation in the Sun.

DOI: 10.1103/PhysRevD.81.057101

In a recent work [1], we presented the result of a search for neutralino dark matter accumulated in the center of the Sun with the 22-string configuration of the IceCube detector. In this paper we extend the search to an alternative dark matter candidate, Kaluza-Klein (KK) particles, arising from theories with extra spacetime dimensions. In the simplest framework of universal extra dimensions (UED) [2], there is a single extra dimension of size $R \sim$ $\mathcal{O}\left(\mathrm{TeV}^{-1}\right)$ compactified on an $S^{1} / Z_{2}$ orbifold. Within minimal UED theories, the first excitation of the hypercharge gauge boson, $B^{(1)}$, is generally the lightest KaluzaKlein particle (LKP). It is often denoted as the KK "photon," $\gamma^{(1)}$, since the effective first KK-level Weinberg angle of the mass matrix is very small, and therefore $B^{(1)}$ can also be described as a mass eigenstate [2]. KK-parity conser-

\footnotetext{
*Corresponding author: danning@fysik.su.se (M. Danninger). ${ }^{\dagger}$ Affiliated with Universität Erlangen-Nürnberg, Physikalisches Institut, D-91058, Erlangen, Germany

On leave of absence from Università di Bari and Sezione INFN, Dipartimento di Fisica, I-70126, Bari, Italy

${ }^{\S}$ Affiliated with School of Pure and Applied Natural Sciences, Kalmar University, S-39182 Kalmar, Sweden

"Corresponding author: wikstrom@fysik.su.se (G. Wikström).
}

PACS numbers: 95.35.+d, 14.70.Pw, 96.50.S-, 98.70.Sa

vation, affiliated with extra-dimensional momentum conservation, leads to the stability of the LKP, which makes it a viable dark matter (DM) candidate. There are also other possible natural choices for LKP candidates within UED, like the KK "graviton,", the KK "neutrino" or the $Z^{(1)}$ boson that may constitute viable DM candidates. They are not considered here. Instead, we focus on the most promising KKDM prospect in terms of indirect detection expectations, the KK "photon." Accelerator measurements constrain the lower bound for the mass of the LKP, $m_{\gamma^{(1)}}$, at $300 \mathrm{GeV}$ [3]. The upper bound is limited to a few $\mathrm{TeV}$ in order to not exceed the observed DM relic density and over close the Universe. Within this mass range, the relic density of LKPs is in the favorable region for providing the cold dark matter of the Universe, as observed by [4]. LKPs are assumed to have been in thermal equilibrium in the early Universe and therefore their relic density depends on the LKP mass, the annihilation cross section and the coannihilation rate. The annihilation and coannihilation cross sections are determined by the mass spectrum of the first KK level and standard model couplings [5]. We here consider UED models with five spacetime dimensions characterized by two parameters: the LKP mass, $m_{\gamma^{(1)}}$, and the 
TABLE I. LKP annihilation branching ratios for two values of $\Delta_{q^{(1)}}$ [8]. Ratios are not summed over generations. Channels within parenthesis give negligible contribution to a neutrino flux from the Sun. The Higgs-field annihilation channel, marked with $\dagger$, is neglected, due to large uncertainty and small contribution to the neutrino flux.

\begin{tabular}{lcc}
\hline \hline Channel & \multicolumn{2}{c}{ Branching ratio } \\
\hline & $\Delta_{q^{(1)}}=0$ & $\Delta_{q^{(1)}}=0.14$ \\
$\left(e^{+} e^{-}\right),\left(\mu^{+} \mu^{-}\right), \tau^{+} \tau^{-}$ & 0.20 & 0.23 \\
$(u \bar{u}), c \bar{c}, t \bar{t}$ & 0.11 & 0.077 \\
$(d \bar{d}),(s \bar{s}), b \bar{s}$ & 0.007 & 0.005 \\
$\nu_{e} \bar{\nu}_{e}, \nu_{\mu} \bar{\nu}_{\mu}, \nu_{\tau} \bar{\nu}_{\tau}$ & 0.012 & 0.014 \\
$\left(\Phi, \Phi^{*}\right)^{\dagger}$ & 0.023 & 0.027 \\
\hline \hline
\end{tabular}

mass splitting $\Delta_{q^{(1)}} \equiv\left(m_{q^{(1)}}-m_{\gamma^{(1)}}\right) / m_{\gamma^{(1)}}$, where $m_{q^{(1)}}$ is the mass of the first KK quark excitation, as discussed in [2,5-7]. As a possible dark matter component of the halo, LKPs can become gravitationally trapped in massive celestial bodies like the Sun, accumulating in the object's core. Since the LKP is a boson, pairwise annihilation is dominated by $s$-wave processes, creating standard model particles whose decay chains produce neutrinos in the $\mathrm{GeV}-\mathrm{TeV}$ range. The branching ratios for the LKP annihilation channels of interest are given in Table I for two values of $\Delta_{q^{(1)}}$ [8]. The neutrinos may escape the Sun and reach Earth. The search presented here aims at detecting LKP annihilations indirectly by observing an excess of such high energy neutrinos from the direction of the Sun. Despite the existence of various limits on neutralino induced neutrino fluxes from the Sun [1,9-12], no corresponding limits for LKP annihilations have been previously reported.

For the results presented here, we use the same data set, 104.3 days live time taken with the 22-string configuration of IceCube in 2007, and the same analysis cuts as presented in [1]. This is justified since the signature of the expected signal at the detector is very similar for the LKP and neutralinos, considering the hardest $\tilde{\chi}_{1}^{0}$-annihilation channel into $W^{+} W^{-}$. The neutrino spectrum from annihilations of a LKP of a given mass in the center of the Sun is considerably harder than that of a neutralino of the same mass. However, oscillations and energy losses of the neutrinos on their way out of the Sun, like neutral current scattering, absorption, and $\nu_{\tau}$ regeneration, smear out the energy spectra in a way that makes them comparable at Earth. Figure 1 shows an example of how the resulting muon spectra at the detector compare for a selected choice of neutralino and LKP masses at 250 and $3000 \mathrm{GeV}$. The analysis strategy used in [1] is therefore already optimized for the search of KK dark matter.

We simulated LKP annihilations in the Sun using WIMPSIM [13] for LKP masses $m_{\gamma^{(1)}}=250,500,700$, $900,1100,1500,3000 \mathrm{GeV}$. We used $\Delta_{q^{(1)}}=0$ with annihilation branching ratios from Table I. Since $\Delta_{q^{(1)}}>0$

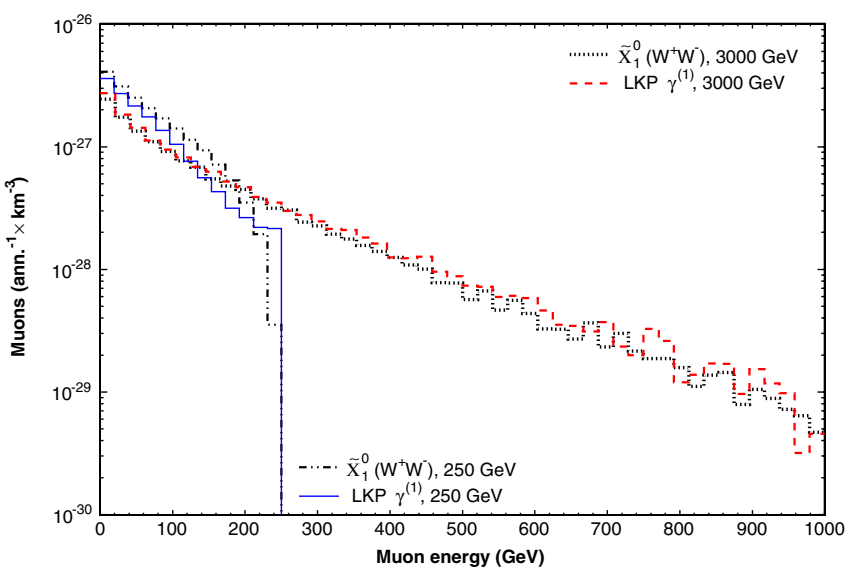

FIG. 1 (color online). Comparison of simulated muon spectra from LKP, $\gamma^{(1)}$, and neutralino, $\tilde{\chi}_{1}^{0}$, annihilations observed in IceCube, for two WIMP masses, 250 and $3000 \mathrm{GeV}$, representing the boundaries of the investigated LKP model space.

results in an increased neutrino flux due to the importance of the contributions from the $\tau^{+} \tau^{-}$and the direct neutrino channels, the choice of $\Delta_{q^{(1)}}=0$ leads to a conservative limit. The background in the search for neutrinos from the Sun comes from air showers created in cosmic ray interactions in the atmosphere. The showers cause downward going atmospheric muon events, triggering the detector at $\sim 500 \mathrm{~Hz}$, and atmospheric muon neutrino events, triggering at $\sim 4 \mathrm{mHz}$. When the Sun is below the horizon, the neutrino signal can be distinguished from the atmospheric muon background by selecting events with upward-going reconstructed tracks.

Atmospheric muon and neutrino background events were also generated $[14,15]$. The propagation of muons and photons in the ice was simulated $[16,17]$ taking measured ice properties into account [18].

The events had to pass several selection criteria as described in [1] in order to reduce the content of atmospheric muon events. As a compromise between signal efficiency and background rejection, it was required that more than half of the events in the final data sample were neutrino induced. The observables used describe the quality of the track reconstructions and the geometry and time evolution of the hit pattern in the detector, and they were required to be well reproduced in simulations. The event selection consisted first in a series of unidimensional cuts on the selected event variables, and a final step that used two support vector machines (SVM). The SVMs were trained with simulated signal, and a set of experimental data, recorded in December 2007 and not used in this analysis since the Sun was above the horizon, was taken as background. A final sample was then defined from a cut on the combined two SVM output values, $Q_{1} \times Q_{2}$ (see Fig. 1 in [1]). The analysis was performed in a blind manner such that the azimuth of the Sun is unknown until the selection criteria were finalized. 
The systematic uncertainties on the effective volume, $V_{\text {eff }}$, defined as the equivalent detector volume with $100 \%$ selection efficiency, are the same as the ones calculated in the WIMP analysis in [1], and are dominated by the uncertainties in photon propagation in the ice and the absolute digital optical module efficiency. They range from $\pm 19 \%$ for the highest $m_{\gamma^{(1)}}$ to $\pm 26 \%$ for the lowest $m_{\gamma^{(1)}}$ [19]. From the final event selection of the signal simulation we additionally derive the effective area for muon neutrinos from the direction of the Sun as a function of neutrino energy, see Fig. 2. Also shown in the figure is the median angular error, the median of the angle between the reconstructed muon and the neutrino direction, $\Theta$. The result includes systematic uncertainties and is an average over the austral winter, during which the Sun is below the horizon.

For the LKP signal models we then calculated the effective volume and, based on the distribution of the reconstructed angle to the Sun $\Psi$, we constructed confidence intervals at the $90 \%$ confidence level using the method outlined in [1]: to evaluate the signal content in the final event sample, hypothesis testing was done based on $\Psi$, the angle between the reconstructed track and the direction of the Sun. From simulations we find $f_{s}(\Psi)$, the probability distribution of $\Psi$ for the signal. By randomizing the azimuth angle in the final event sample of experimental data, $f_{b}(\Psi)$, the equivalent probability distribution is found for background. Defining $\xi=\frac{\mu_{s}}{n_{\mathrm{obs}}}$, from the number of signal events $\mu_{s}$ and the observed number of events $n_{\mathrm{obs}}$, we form the combined probability density $f_{\xi}(\Psi)=\xi \cdot f_{s}(\Psi)+$ $(1-\xi) \cdot f_{b}(\Psi)$. Based on $\xi_{\text {best }}$, the non-negative signal

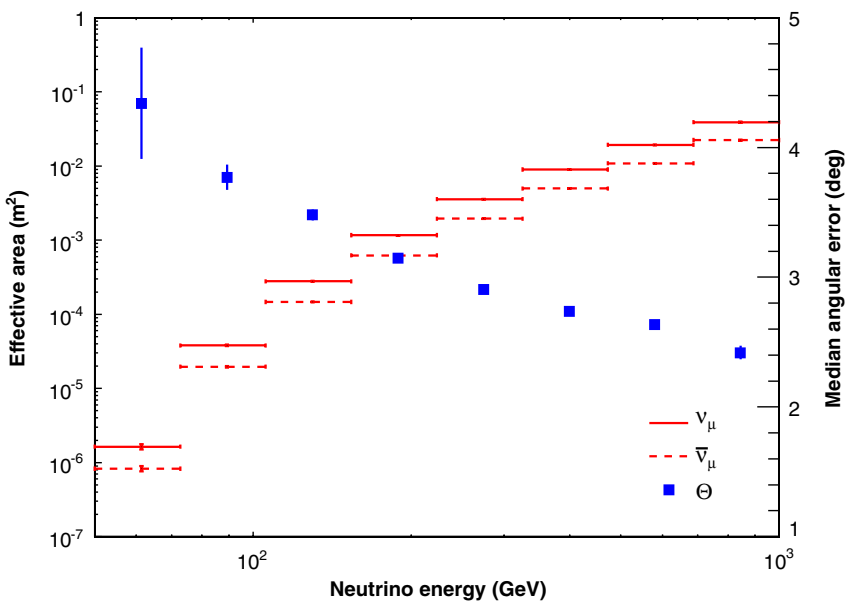

FIG. 2 (color online). Lines showing the effective area (left scale) for the final event selection as function of neutrino energy in the range $50-1000 \mathrm{GeV}$, for muon neutrinos (solid line) and antineutrinos (dashed line) from the direction of the Sun. The result is an average over the austral winter. Systematic effects are included at the $1 \sigma$ level, and statistical uncertainty of the same level are shown with error bars. Also shown is the median angular error $\Theta$ with $1 \sigma$ error bars (squares, right scale). content that maximizes the likelihood, we form the logarithm of the likelihood ratio $R(\xi)=\log \left(\prod_{i=1}^{i=n_{\text {obs }}} \frac{f_{\xi}\left(\Psi_{i}\right)}{f_{\xi_{\text {best }}}\left(\Psi_{i}\right)}\right)$ [20]. Comparing this with a $R_{\text {test }}(\xi)$ distribution of a large number of pseudoexperiments with $n_{\text {obs }}$ events taken from $f_{\xi}(\Psi)$, we construct the confidence interval on $\xi$ at significance $\alpha$ as $R\left(\xi_{\text {lim }}\right)=R_{\text {test }}^{\alpha}\left(\xi_{\text {lim }}\right)$, where $P\left(R_{\text {test }}>\right.$ $\left.R_{\text {test }}^{\alpha}\right)=1-\alpha$.

No excess of events from the Sun above the background expectation was found in the search $\left(\xi_{\text {best }}=0\right)$. The observed number of events as a function of the angle to the Sun, $\Psi$, is compared to the atmospheric background expectation in Fig. 3. From the upper limits on the number of signal events $\mu_{s}$ we calculate the limit on the neutrino to muon conversion rate $\Gamma_{\nu \rightarrow \mu}=\frac{\mu_{s}}{V_{\text {eff }} \cdot t}$, for the live time $t$. Using the signal simulation [13], we can convert this rate to a limit on the LKP annihilation rate in the Sun, $\Gamma_{\mathrm{A}}$, see Table II. Results from different experiments are commonly compared by calculating the limit on the muon flux above $1 \mathrm{GeV}, \Phi_{\mu}$, which is also given in Table II together with the sensitivity, $\bar{\Phi}_{\mu}$, the median limit obtained from simulations with no signal.

The flux limit is shown in Fig. 4 together with the theoretically allowed flux region, derived from Refs. [6,8] with the use of Dark SUSY [21]. We have here approximated the branching ratios for the regions of $\Delta_{q^{(1)}}=0.01$ and $\Delta_{q^{(1)}}=0.1$ with those of $\Delta_{q^{(1)}}=0$ and $\Delta_{q^{(1)}}=0.14$, respectively, as given in Table I. The limits on the annihilation rate can be converted into limits on the spin-dependent, $\sigma^{\mathrm{SD}}$, and spin-independent, $\sigma^{\mathrm{SI}}$, LKPproton cross sections, allowing a comparison with the results of direct search experiments. Since capture in the

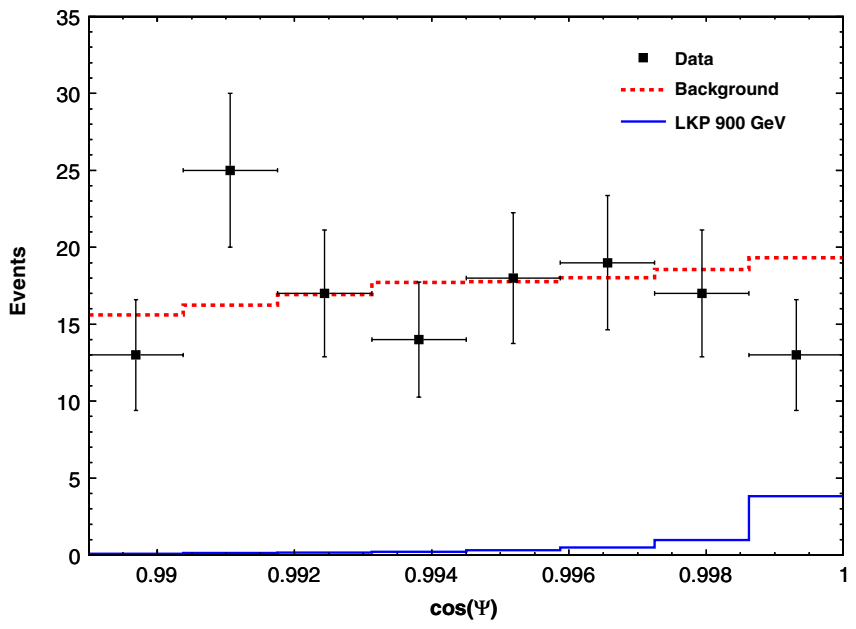

FIG. 3 (color online). Cosine of the angle between the reconstructed track and the direction of the Sun, $\Psi$, for data (squares) with 1 standard deviation error bars, and the atmospheric background expectation from atmospheric muons and neutrinos (dashed line). Also shown is a simulated signal $\left(m_{\gamma^{(1)}}=\right.$ $900 \mathrm{GeV}$ ) scaled to $\mu_{s}=7.0$ events (see Table II). 
TABLE II. Upper limits on the number of signal events $\mu_{s}$, the conversion rate $\Gamma_{\nu \rightarrow \mu}$, the LKP annihilation rate in the Sun $\Gamma_{\mathrm{A}}$, the muon flux $\Phi_{\mu}$, and the LKP-proton scattering cross sections (spin independent, $\sigma^{\mathrm{SI}}$, and spin dependent, $\sigma^{\mathrm{SD}}$ ), at the $90 \%$ confidence level, including systematic errors. The sensitivity $\bar{\Phi}_{\mu}$ (see text) is shown for comparison. Also shown is the median angular error $\Theta$, the mean muon energy $\left\langle E_{\mu}\right\rangle$, the effective volume $V_{\text {eff }}$, and the $\nu_{\mu}$ effective area $A_{\text {eff }}$.

\begin{tabular}{|c|c|c|c|c|c|c|c|c|c|c|c|}
\hline $\begin{array}{l}m_{\gamma^{(1)}} \\
(\mathrm{GeV})\end{array}$ & $\mu_{s}$ & $\begin{array}{c}\Gamma_{\nu \rightarrow \mu} \\
\left(\mathrm{km}^{-3} \mathrm{y}^{-1}\right)\end{array}$ & $\begin{array}{c}\Gamma_{\mathrm{A}} \\
\left(\mathrm{s}^{-1}\right)\end{array}$ & $\begin{array}{c}\Phi_{\mu} \\
\left(\mathrm{km}^{-2} \mathrm{y}^{-1}\right)\end{array}$ & $\begin{array}{c}\bar{\Phi}_{\mu} \\
\left(\mathrm{km}^{-2} \mathrm{y}^{-1}\right)\end{array}$ & $\begin{array}{c}\sigma^{\mathrm{SI}} \\
\left(\mathrm{cm}^{2}\right)\end{array}$ & $\begin{array}{c}\sigma^{\mathrm{SD}} \\
\left(\mathrm{cm}^{2}\right)\end{array}$ & $\begin{array}{l}\Theta \\
\left({ }^{\circ}\right)\end{array}$ & $\begin{array}{c}\left\langle E_{\mu}\right\rangle \\
(\mathrm{GeV})\end{array}$ & $\begin{array}{c}V_{\text {eff }} \\
\left(\mathrm{km}^{3}\right)\end{array}$ & $\begin{array}{l}A_{\text {eff }} \\
\left(\mathrm{m}^{2}\right)\end{array}$ \\
\hline 250 & 7.2 & $3.3 \cdot 10^{3}$ & $7.9 \cdot 10^{21}$ & $8.7 \cdot 10^{2}$ & $1.7 \cdot 10^{3}$ & $4.9 \cdot 10^{-43}$ & $3.7 \cdot 10^{-40}$ & 3.2 & 65.8 & $7.6 \cdot 10^{-3}$ & $1.1 \cdot 10^{-4}$ \\
\hline 500 & 6.9 & $1.2 \cdot 10^{3}$ & $2.2 \cdot 10^{21}$ & $4.6 \cdot 10^{2}$ & $8.8 \cdot 10^{2}$ & $4.1 \cdot 10^{-43}$ & $4.1 \cdot 10^{-40}$ & 3.0 & 103 & $2.1 \cdot 10^{-2}$ & $4.0 \cdot 10^{-4}$ \\
\hline 700 & 7.3 & $9.2 \cdot 10^{2}$ & $1.7 \cdot 10^{21}$ & $4.1 \cdot 10^{2}$ & $7.1 \cdot 10^{2}$ & $5.6 \cdot 10^{-43}$ & $6.2 \cdot 10^{-40}$ & 2.9 & 122 & $2.8 \cdot 10^{-2}$ & $5.7 \cdot 10^{-4}$ \\
\hline 900 & 7.0 & $7.8 \cdot 10^{2}$ & $1.5 \cdot 10^{21}$ & $3.8 \cdot 10^{2}$ & $6.6 \cdot 10^{2}$ & $7.3 \cdot 10^{-43}$ & $8.6 \cdot 10^{-40}$ & 2.9 & 134 & $3.2 \cdot 10^{-2}$ & $6.6 \cdot 10^{-4}$ \\
\hline 1100 & 7.2 & $7.6 \cdot 10^{2}$ & $1.5 \cdot 10^{21}$ & $3.8 \cdot 10^{2}$ & $6.6 \cdot 10^{2}$ & $1.0 \cdot 10^{-42}$ & $1.3 \cdot 10^{-39}$ & 2.9 & 141 & $3.3 \cdot 10^{-2}$ & $7.0 \cdot 10^{-4}$ \\
\hline 1500 & 7.2 & $6.6 \cdot 10^{2}$ & $1.3 \cdot 10^{21}$ & $3.5 \cdot 10^{2}$ & $6.0 \cdot 10^{2}$ & $1.7 \cdot 10^{-42}$ & $2.2 \cdot 10^{-39}$ & 2.9 & 151 & $3.8 \cdot 10^{-2}$ & $7.9 \cdot 10^{-4}$ \\
\hline 3000 & 6.7 & $6.2 \cdot 10^{2}$ & $1.5 \cdot 10^{21}$ & $3.3 \cdot 10^{2}$ & $5.8 \cdot 10^{2}$ & $7.4 \cdot 10^{-42}$ & $9.9 \cdot 10^{-39}$ & 2.8 & 152 & $3.8 \cdot 10^{-2}$ & $7.0 \cdot 10^{-4}$ \\
\hline
\end{tabular}

Sun is dominated by $\sigma^{\mathrm{SD}}$, indirect searches are expected to be competitive in setting limits on this quantity. Assuming equilibrium between the capture and annihilation rates in the Sun, the annihilation rate is directly proportional to the cross section. A conservative limit on $\sigma^{\mathrm{SD}}$ is found by setting $\sigma^{\mathrm{SI}}$ to zero, and vice versa. We have used the method described in Ref. [22] to perform the conversion. The results are shown in Table II. We assumed a local WIMP density of $0.3 \mathrm{GeV} / \mathrm{cm}^{3}$, and a Maxwellian WIMP velocity distribution with a dispersion of $270 \mathrm{~km} / \mathrm{s}$. Planetary effects on the capture were neglected [23]. Figure 5 shows the limits on $\sigma^{\mathrm{SD}}$, as obtained with the 22-string configuration of IceCube compared with other bounds [24-26], and the KK model space. The theoretical model space (green area) is plotted for different predictions for the mass splitting $\Delta_{q^{(1)}}$. The blue regions indicate the overlap regions with two different $\Omega_{\mathrm{CDM}}$ intervals,

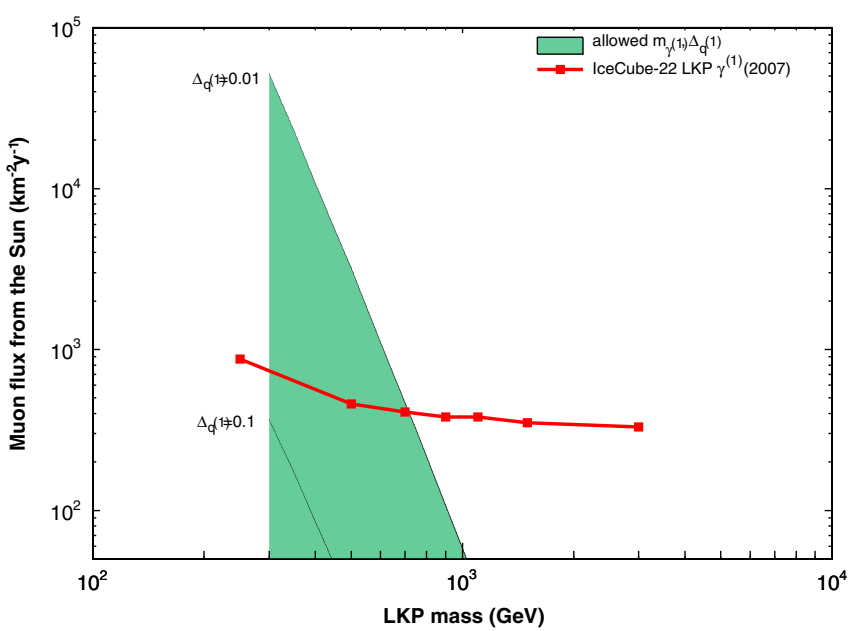

FIG. 4 (color online). Limits on the muon flux from LKP annihilations in the Sun including systematic errors (squares), compared to the theoretically allowed region of $m_{\gamma^{(1)}}$ and $\Delta_{q^{(1)}}$. The regions corresponding to $\Delta_{q^{(1)}}=0.01$ and $\Delta_{q^{(1)}}=0.1$ are marked with black lines. The region below $m_{\gamma^{(1)}}=300 \mathrm{GeV}$ is excluded by collider experiments [3]. whereas the narrow dark blue region corresponds to the preferred WMAP $1 \sigma$ region for cold dark matter. The upper bound on $m_{\gamma^{(1)}}$, derived from the overclosure limit for each individual LKP model [5], varies with different values of $\Delta_{q^{(1)}}$ and increases remarkably for models with $\Delta_{q^{(1)}}<0.1$. This is due to additional coannihilation effects, arising for degenerate LKP models [6].

In conclusion, we have presented the first limits on LKP annihilations in the Sun. We also derived the most stringent limits on the spin-dependent LKP-proton cross sections in the nonexcluded LKP mass regions $\left(300 \mathrm{GeV}<m_{\gamma^{(1)}}<\right.$ $3 \mathrm{TeV}$ ), improving existing limits by more than 2 orders of

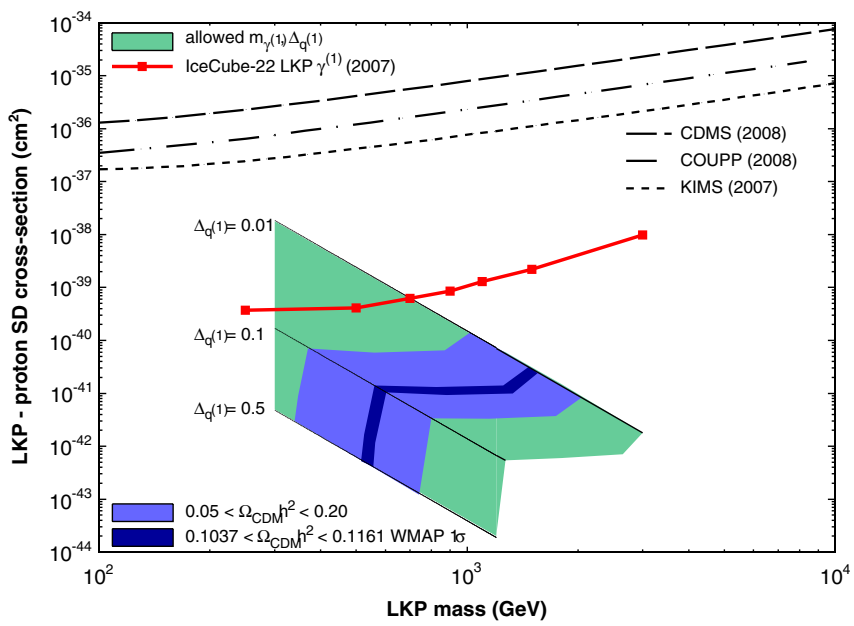

FIG. 5 (color online). Limits on the LKP-proton SD scattering cross section (squares) adjusted for systematic effects, compared with limits from direct detection experiments [24-26]. Theoretically predicted cross sections are indicated by the green area [6]. The regions corresponding to $\Delta_{q^{(1)}}=0.01,0.1,0.5$ are marked with black lines. The region below $m_{\gamma^{(1)}}=300 \mathrm{GeV}$ is excluded by collider experiments [3] and the upper bound on $m_{\gamma^{(1)}}$ corresponds to the overclosure limit for each individual LKP model [5]. The lighter blue region is allowed when considering $0.05<\Omega_{\mathrm{CDM}} h^{2}<0.20$, and the darker blue region corresponds to the preferred $1 \sigma$ WMAP $(5 \mathrm{yr}$ ) relic density $0.1037<\Omega_{\mathrm{CDM}} h^{2}<0.1161$ [4]. 
magnitude and excluding some viable LKP models. The full IceCube detector with the DeepCore extension [27] is expected to test most LKP models within the allowed region for $0.05<\Omega_{\mathrm{CDM}} h^{2}<0.20$, shown in Fig. 5 .

We thank the following agencies: U.S. National Science Foundation-Office of Polar Programs, U.S. National Science Foundation-Physics Division, University of Wisconsin Alumni Research Foundation, U.S. Department of Energy, NERSC, the LONI grid; Swedish
Research Council, K.\&A. Wallenberg Foundation, Sweden; German Ministry for Education and Research, Deutsche Forschungsgemeinschaft; Fund for Scientific Research, IWT-Flanders, BELSPO, Belgium; the Netherlands Organisation for Scientific Research; M. Ribordy is supported by SNF (Switzerland); A. Kappes and A. Groß are supported by the EU Marie Curie OIF Program. We thank S. Arrenberg and K. Kong for helpful correspondence and details on their paper.
[1] R. Abbasi et al., Phys. Rev. Lett. 102, 201302 (2009).

[2] D. Hooper and S. Profumo, Phys. Rep. 453, 29 (2007).

[3] J. F. Oliver et al., Phys. Rev. D 67, 056002 (2003).

[4] J. Dunkley et al., Astrophys. J. Suppl. Ser. 180, 306 (2009).

[5] G. Servant and T.M. Tait, Nucl. Phys. B650, 391 (2003).

[6] S. Arrenberg et al., Phys. Rev. D 78, 056002 (2008).

[7] H. C. Cheng and J. L. Feng, K. T. Matchev, Phys. Rev. Lett. 89, 211301 (2002).

[8] D. Hooper and G. D. Kribs, Phys. Rev. D 67, 055003 (2003).

[9] M. M. Boliev et al., Nucl. Phys. B, Proc. Suppl. 48, 83 (1996).

[10] M. Ambrosio et al., Phys. Rev. D 60, 082002 (1999).

[11] S. Desai et al., Phys. Rev. D 70, 083523 (2004).

[12] M. Ackermann et al., Astropart. Phys. 24, 459 (2006).

[13] M. Blennow, J. Edsjö, and T. Ohlsson, J. Cosmol. Astropart. Phys. 01 (2008) 021.

[14] D. Heck et al., FZKA, Report No. 6019, 1998.
[15] A. Gazizov and M. Kowalski, Comput. Phys. Commun. 172, 203 (2005).

[16] D. Chirkin and W. Rhode, arXiv:hep-ph/0407075v2.

[17] J. Lundberg et al., Nucl. Instrum. Methods Phys. Res., Sect. A 581, 619 (2007).

[18] M. Ackermann et al., J. Geophys. Res. 111, D13203 (2006).

[19] G. Wikström, PhD thesis, Stockholm University, 2009.

[20] G. J. Feldman and R. D. Cousins, Phys. Rev. D 57, 3873 (1998).

[21] P. Gondolo et al., J. Cosmol. Astropart. Phys. 07 (2004) 008.

[22] G. Wikström and J. Edsjö, J. Cosmol. Astropart. Phys. 04 (2009) 009.

[23] A. H. G. Peter, Phys. Rev. D 79, 103532 (2009).

[24] Z. Ahmed et al., Phys. Rev. Lett. 102, 011301 (2009).

[25] H. S. Lee et al., Phys. Rev. Lett. 99, 091301 (2007).

[26] E. Behnke et al., Science 319, 933 (2008).

[27] E. Resconi for the (IceCube Collaboration), Nucl. Instrum. Methods Phys. Res., Sect. A 602, 7 (2009). 